\title{
Evaluation of the repeatability and reproducibility of small-scale fermentation methodology for Tempranillo and Cabernet Sauvignon
}

\author{
Antoni Sánchez-Ortiz ${ }^{1}$ \\ ${ }^{1}$ Affiliation not available
}

June 11, 2020

\begin{abstract}
Researchers typically perform winemaking experiments using small amounts of grapes. Few studies have actually evaluated both the repeatability and reproducibility of small-scale fermentations in Tempranillo and Cabernet Sauvignon. This study examines, for two different phenolic potential varieties, the repeatability and reproducibility of the small-scale red winemaking in 4 tank sizes $(10,25,50$ and $100 \mathrm{~L})$, and how volume size may affect to the composition of the wine. Additionally, for each variety we carried out a commercial fermentation using a $2500 \mathrm{~L}$ tank. Medium size-tanks gave the best extraction for the phenolic composition. High repeatability was observed for alcohol content, $\mathrm{pH}$ and total acidity, anthocyanins and procyanidins for both varieties. This research gives a solid basis for validating the reproducibility of the small-scale fermentations of two different phenolic potential red grapevines andsheds new light on small-scale winemaking potentiality and limitations.
\end{abstract}

\section{Introduction}

Research in viticulture mostly relies on measuring yield and grape composition to assess which cultural practices convey an improvement in vine performance and, as a consequence, could be worth implementing in field. This approach, though useful, does not allow a completely satisfactory evaluation, since researchers cannot assess to which extent the effects observed are transferred to wine composition, i.e.: to final product quality.

In order to overcome that limitation, some authors introduce small-scale fermentations in their experiments to obtain a more complete evaluation, which is widely recognized as a step-forward in research applicability. However, despite its relevance, little attention has been paid to evaluate to which extent reducing grape volume in small-scale winemaking, affects fermentation dynamics, wine composition and reproducibility. On the one side, some authors such as ${ }^{1}$, Dallas et al (2003) and ${ }^{2}$ González-Manzano et al. (2003) published results on grape seed extraction in model wine solutions, whereas other authors such as ${ }^{3}$ and Rossouw et al. (2012) studied yeast performance as influenced by commercial and small-scaled tanks. ${ }^{4}$ also studied the changes occurring in grapes after frozing the samples to check the lack of repeatability between fresch and frozen grapes. On the other side, as direct comparison of small-scale to commercial winemaking, ${ }^{5}$ compared the aromatic compounds of Chardonnay, and only three experiments used different fermentation volumes in red varieties. ${ }^{6}$ compared three wine volumes $(20,50$ and $300 \mathrm{~kg}$ ) of a blend with Cabernet Sauvignon and Cabernet Franc), in an experiment that was focused on evaluating the suitability of frozen must, to finally report that winemaking outcomes were comparable among the three volumes compared. The same research team, Schmid and Jiranek (2011), compared fresh, frozen and blast-frozen grapes fermentation using two different volumes $(80$ and $500 \mathrm{~kg}$ ), concluding that wines were similar in winetasting. Last, Sampaio et 
al. (2007) compared Pinot noir in very small-volume $(3.5 \mathrm{~kg})$ to a commercial fermentation $(4540 \mathrm{~kg})$, and observed that it was possible to effectively control oxidation and spoilage at this volume, although significant differences were observed in wine composition between both scales. ${ }^{7}$

Therefore, the existing research in this field is scarce. Thus, taking into account that small-scale winemaking conditions vary between experiments, as regional or winemaker preferences and protocol modifications may affect any stage of winemaking (i.e., yeast inoculation, cap management regime and malolactic fermentation), there is a clear need of learning how winemaking conditions and, particularly, tank size, affect the composition of the wines obtained. Moreover, the above mentioned research did not pay attention to repeatability, which is particularly relevant since, if high variability showed up, the interest of small-scale winemaking would be limited, as far as an additional source of variation could interfere with data analysis.

The aim of this work is to evaluate how repeatable and reproducible small-scale winemaking is. The differential aspect of this research is that 4 replicates are used with 4 different volumes in 2 distinct red varieties, and that the small-scale fermentation protocols used mirrored typical winemaking techniques commonly used in small wineries producing premium red wines worldwide.

\section{Materials and Methods}

\section{Experimental design}

This research was conducted in the experimental winery of the Enology Faculty in Tarragona, Spain, using

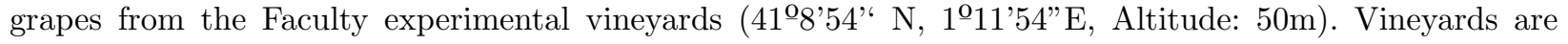
located near the coast in the Designation of Origin Tarragona (Spain), which has a Mediterranean climate, soils are typically fertile and dense, and were managed according to standard practices in the region. Grapes from two distinct varieties were used, Tempranillo (TE) and Cabernet Sauvignon (CS), the former showing large berries and low-to-medium phenolic potential, and the latter small sized berries and high phenolic content. For both varieties, four different small-scale volumes (10, 25, 50 and $100 \mathrm{~L})$ were compared. All vessels have a ratio height/diameter ranging between 1.4-1.5. All the tanks were made of stainless-steel, with a rubber gasket that helped to keep the lid tight. For each variety and tank volume, four replicates were vinified. Additionally, a commercial-sized large fermentation was performed in a $2500 \mathrm{~L}$ stainless steel tank.

\section{Winemaking procedure}

Grapes were handpicked at full ripeness into $20 \mathrm{~kg}$ boxes, and stored at $21^{\circ} \mathrm{C}$ in a cold room before crushing. Grapes were de-stemmed and crushed individually for each tank volume using a Bücher Delta E2. Tanks were filled one by one $3 / 4 \mathrm{~s}$ full in order to ensure an upper appropriate fermentation cap management. Room temperature during fermentation was kept at $23^{\circ} \mathrm{C} ; 40 \mathrm{mg} \mathrm{L}^{-1}$ sulfur dioxide was added to the must. All

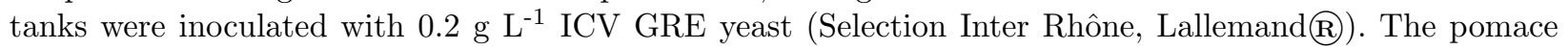
was gently hand punched down twice a day until alcoholic fermentation was accomplished. During the tumultuous stage, must density and temperature were both measured daily, controlling sugar consumption and avoiding extremely high temperatures (higher than $28^{\circ} \mathrm{C}$ ) during the winemaking process. The pomace was pressed once fermentation was completely exhausted (reducing sugars $<2 \mathrm{~g} \mathrm{~L}^{-1}$ ), which took 8 days in TE and 12 in CS. Free run wines were then obtained by using a cone-shaped funnel (Lacor inox 18/20diameter $22 \mathrm{~cm}$ ) to separate the pomace from the wine. Press wine was obtained using a 40L Hydropress with a capacity of juice yield of up to 20-25 liters per pressing, depending on variety and ripeness of fruit (http://www.vigopresses.co.uk). After pressing, the juice was settled overnight and racked to the same tank to promote clarity. Potassium metabisulphite was added (Winy Sepsa Enartis) to reach $20 \mathrm{mg} \mathrm{L}^{-1}$ of Sulphur dioxide to prevent microbial spoilage. Wines were stabilized at $4{ }^{\circ} \mathrm{C}$ for 2 months, followed by racking before bottling in December, and kept at $4^{\mathrm{O}} \mathrm{C}$ for further storage. Finished wines were bottled without fining or filtering. The wines did not undergo malolactic fermentation to avoid unwanted apparent malolactic deviations, and no oak or ageing was made. 


\section{Grape analysis}

All grape batches were analyzed before they were introduced in each tank. 100-berries from each variety were used to determine the sugar level, acidity and $\mathrm{pH}$; another 300 berries were used to analyze phenolic maturity. Sugar content was determined using a handheld portable refractometer (Model 102/112/102bp). Titratable acidity (TA, g/L) was measured by titration with sodium hydroxide, and $\mathrm{pH}$ measured using a pH-meter (Crison Micro CM 2201). The modified Glories method, consisting of berry samples macerated at $\mathrm{pH} 3.6$ instead of $\mathrm{pH} 3.2^{8}$ was used to analyze phenolic maturity. Berries were blended (Oster Blender Classic 3 Model 4655) and macerated in an agitator Edmund Bühler GmBH SM-30, to determine total anthocyanins (TAnt) (Ribéreau-Gayon et al. 2000) and tannins (Ribéreau-Gayon et Stonestreet, 1965).

Wine analysis

$\mathrm{ABV}, \mathrm{pH}, \mathrm{TA}$, TAnt and tannins within the tank-size was analyzed. Anthocyanin contents were determined following the methodology detailed in Valls et al. (2009) through HPLC using a Hewlett Packard Liquid Chromatograph (Waters Corporation, Mildford, MA, USA) equipped with a Zorbax Eclipse Plus C18 Column $(150 \times 2.1 \mathrm{~mm} 3.5 \mu \mathrm{m})$ and a Zorbax Eclipse Plus-C18 Precolumn $(12.5 \times 4.6 \mathrm{~mm} 5 \mu \mathrm{m})$. Injection volume was 5 $\mu \mathrm{L}$; elution was performed with a mobile phase A of water HPLC-grade $(0.2 \%$ trifluoroacetic acid $)$ and a mobile phase B using methanol (0.2\% trifluoroacetic acid). Column temperature was set at $50{ }^{\circ} \mathrm{C}$. The HPLC was coupled to a Diode Array Detector (DAD). Quantifications were performed using the DAD detector, and identifications were made considering the TOF (time of flight). Mass spectrometry (MS) detector was used to assist for the identification. Content of free anthocyanins was determined using a calibration curve (based on peak area, $\mathrm{y}=0.7968 \mathrm{x}+7.5756, \mathrm{R}^{2}=0.9774$ ), which was established using malvidin 3 -glucoside standard solutions submitted to the same procedure. The anthocyanidin-3-monoglucosides and respective acetylated and coumaroylated glycosides were identified on the basis of their UV-vis spectra and retention times (Table 1). The anthocyanidins were identified by HPLC by comparison with internal standards. Calibration curves were obtained by injecting standards with different concentrations of malvidin 3-glucoside (Extrasynthese, Genay, France). The range of the linear calibration curves was 0.1 to $1.0 \mathrm{mg} / \mathrm{L}$ for the lower $\left(\mathrm{R}^{2}>0.996\right), 0.1$ to $5.0 \mathrm{mg} / \mathrm{L}$ for intermediate $\left(\mathrm{R}^{2}>0.987\right)$, and 10.0 to $200.0 \mathrm{mg} / \mathrm{L}$ for the higher concentration compounds $\left(\mathrm{R}^{2}>0.987\right)$. Unknown concentrations were determined from the regression equations and the results were expressed on mg of malvidin 3-glucoside. Repeatability of HPLC analysis gave a coefficient of variation $<$ $7 \%$.

Wine procyanidins were analyzed through RRLC (Rapid Resolution Liquid Chromatography) using a Zorbax Eclipse XDB-C18 50x30 $1.8 \mu \mathrm{m}$. (SFF-C038), RRLC in-line filter, 4.6mm, 0.2um. HPLC Injection volume was $1.4 \mu \mathrm{L}$, flux: $0.7 \mathrm{~mL} / \mathrm{min}$, Mobile phase A: water ( $0.1 \%$ formic acid), Mobile phase B: methanol $(0.1 \%$ formic acid), Column temperature: $30^{\circ} \mathrm{C}$. A Diode Array Detector (DAD) was used for the quantification and the TOFMS (Time of Flight Mass Spectrometer) was used for the identification. Table 2 shows the retention time and $\mathrm{m} / \mathrm{z}$ for each compound.

\section{Data analysis}

The effect of tank size on wine composition was evaluated through one-way ANOVA, and, when $\mathrm{P}<0.05$, Tukey post-hoc test was used. The comparison of small-scale wines to commercial-size tank was done using Principal Component Analysis (PCA), considering 2500 L tank as a supplementary individual, i.e. not including it to calculate the principal components (PC) but evaluating its performance. Statistical analyses were performed using R (R Core Team, 2014, Foundation for Statistical Computing, http://www.R-project.org/), using FactoMineR (Husson et al. 2016) and "factoextra" (Kassambara and Mundt, 2016) packages for PC calculation and graphical representation respectively.

\section{Results}

Grape composition 
Grape composition before fermentation was very similar for all tank sizes (Table 3), and low variability occurred between the tanks of the same size $(\mathrm{CV}<5 \%)$. This fact is essential to guarantee that the differences eventually observed in wine composition were not due to differences in grape composition, but associated to the winemaking process.

\begin{tabular}{ccccccc}
\hline Volume & \multicolumn{2}{c}{ Brix } & $\mathrm{pH}$ & $\mathrm{TA}(\mathrm{g} / \mathrm{L})$ & $\mathrm{Bw}(\mathrm{g})$ \\
\hline TE-10 & $23.1 \pm 0.1^{\mathrm{a}}$ & $3.42 \pm 0.02^{\mathrm{a}}$ & $6.46 \pm 0.12^{\mathrm{a}}$ & $2.21 \pm 0.11^{\mathrm{a}}$ \\
TE-25 & $23.1 \pm 0.1^{\mathrm{a}}$ & $3.40 \pm 0.01^{\mathrm{ab}}$ & $6.53 \pm 0.15^{\mathrm{a}}$ & $2.30 \pm 0.14^{\mathrm{a}}$ \\
TE-50 & $23.2 \pm 0.1^{\mathrm{a}}$ & $3.44 \pm 0.02^{\mathrm{b}}$ & $6.60 \pm 0.10^{\mathrm{a}}$ & $2.20 \pm 0.13^{\mathrm{a}}$ \\
TE-100 & $23.2 \pm 0.1^{\mathrm{a}}$ & $3.41 \pm 0.01^{\mathrm{a}}$ & $6.54 \pm 0.11^{\mathrm{a}}$ & $2.33 \pm 0.08^{\mathrm{a}}$ \\
\hline CS-10 & $23.8 \pm 0.1^{\mathrm{b}}$ & $3.26 \pm 0.02^{\mathrm{b}}$ & $5.00 \pm 0.10^{\mathrm{a}}$ & $1.39 \pm 0.14^{\mathrm{b}}$ \\
CS-25 & $24.1 \pm 0.1^{\mathrm{a}}$ & $3.21 \pm 0.01^{\mathrm{o}}$ & $5.20 \pm 0.10^{\mathrm{a}}$ & $1.43 \pm 0.06^{\mathrm{b}}$ \\
CS-50 & $24.1 \pm 0.1^{\mathrm{a}}$ & $3.26 \pm 0.02^{\mathrm{b}}$ & $5.18 \pm 0.07^{\mathrm{a}}$ & $1.51 \pm 0.12^{\mathrm{b}}$ \\
CS-100 & $23.8 \pm 0.1^{\mathrm{b}}$ & $3.26 \pm 0.01^{\mathrm{b}}$ & $5.21 \pm 0.06^{\mathrm{a}}$ & $1.56 \pm 0.12^{\mathrm{b}}$ \\
\hline
\end{tabular}

Figure 1: Values with different letters within a column denote a statistically significant difference $(\mathrm{p}<0.05)$. Results show the mean value and standard deviation. TA: titratable acidity. Bw: berry weight.

\section{Table 3 Must composition and berry weight of each tank}

\section{Fermentation performance}

Winemaking conditions allowed adequate fermentation dynamics in the 32 tanks included in the experiment, getting a complete transformation sugars into ethanol.

Density rapidly decreased after the second day of fermentation for both varieties, and 5 and 9 days after the start of fermentation, just a small quantity of $\operatorname{sugar}\left(p=1010 \mathrm{~kg} / \mathrm{m}^{3}\right)$ remained in TE and CS respectively. At this point, the second stage of fermentation (slow fermentation process) began and, in 3 days, the remaining sugars were transformed into alcohol.

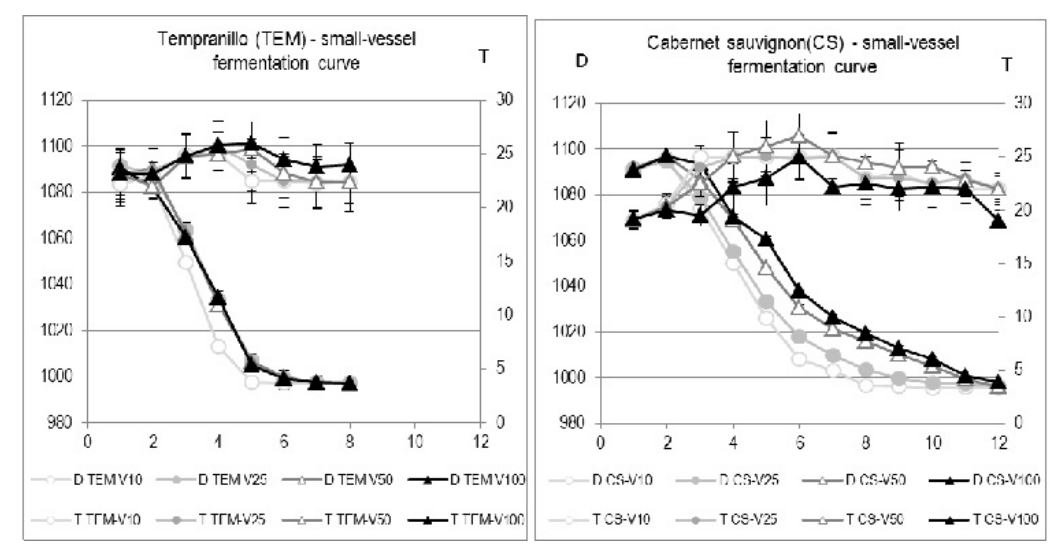

Figure 2: Evolution of fermentation in small-vessels. Evolution of density (D) and temperature (T) during fermentation

In general terms, complete alcoholic fermentation of Tempranillo and Cabernet could be divided into two different stages: tumultuous and slow. The duration of tumultuous fermentation varied according to the composition of the must and the temperature at which it was carried out. Grapes were stored at $21^{\circ} \mathrm{C}$ in a cooler before crushing. Cellar temperature was set at $22^{\circ} \mathrm{C}$ and temperature in the tank was held at $28^{\circ} \mathrm{C}$ at the tumultuous stage to ensure good extraction of polyphenols. This step should be carefully 
considered to avoid uncontrolled fermentations and make this methodology reliable. The yeasts developed comfortably, thus ensuring the total transformation of all sugar into alcohol in both performances. Density rapidly decreased after the second day of fermentation for each variety and vessel (Figure 1).

Approximately 5 and 9 days after the start of fermentation, for Tempranillo and Cabernet respectively, a small quantity of sugar corresponding to a density of $\rho=1010 \mathrm{~kg} / \mathrm{m}^{3}$ remained in the must. At this point, the second stage of fermentation (slow fermentation process) began, which over the following 3 days transformed the remaining final grams of sugar into alcohol. Tempranillo showed a rapid decrease until the $5^{\text {th }}$ day of fermentation when it reached $\rho=997.8,1007.0,1006.5$ and $1005.0 \mathrm{~kg} / \mathrm{m}^{3}$ respectively for each increasing small scale volume $(25,50,75$ and 100L). Fermentation kinetics in Tempranillo required 8 days to ferment all the reducing sugars, showing a slow decrease for the last 3 days. The Cabernet required 12 days to complete the fermentation process. Temperatures never reach more than $28^{\circ} \mathrm{C}$ for both kinetics under the same conditions of controlled room temperature and size vessel. After fermentation, the temperature decreased to $22^{\circ} \mathrm{C}$ in both.

Modelling data using linear functions proved easier to predict the kinetics of the fermentation processes of both varieties/volume studies. As tumultuous fermentation occurred with a different duration for each variety than that of the slow stage, two regression curves were calculated for each combination variety/volume. As expected, tumultuous phase (when maximal fermentation activity occurs) and slow fermentation stage (happening after tumultuous fermentation) clearly show two different slopes (equations are given on Table $3 \mathrm{~b})$.

According to Table 3b, two clear slopes were distinguished on the fermentation curves for both varieties, Tempranillo and Cabernet Sauvignon. Linear regression slopes of tumultuous stage ranged between -21.933 and -24.850 for Tempranillo and -12.286 and -17.321 for Cabernet Sauvignon, indicating a faster kinetic for Tempranillo in the tumultuous fermentation stage. The coefficient of determination is higher in the tumultuous stage. Considering now all volume vessels, Tempranillo slopes from the tumultuous stage would not indicate much different kinetics between volumes, even if $10 \mathrm{~L}$ capacity vessel seems to decrease faster, with a curve done by $\mathrm{y}=-24.8 \mathrm{x}+1121$, than 25,50 and $100 \mathrm{~L}$, which have $\mathrm{y}=-22.4 \mathrm{x}+1124, \mathrm{y}=-22.1 \mathrm{x}+$ 1122 , and $\mathrm{y}=-21.9 \mathrm{x}+1120$, respectively, showing very similar slopes. However, slow stage reveals a similar tendency, having the lowest slope for 10L. Cabernet sauvignon showed a proportional relation between slopes and volume. The 10L volume tank has the higher slope in the tumultuous stage $(\mathrm{y}=-17.3 \mathrm{x}+1119)$ and so the lower slope on the slow stage $(\mathrm{y}=-1.9 \mathrm{x}+1016)$, indicating that the tumultuous part of fermentation proceeds faster in the 10L than any other vessel evaluated.

\section{Effect of small-scale tank volume on wine composition}

With regards to wine composition basic parameters, tank size was observed not to influence ABV, pH and TA neither in CS nor in TE (Table 4), but it affected phenolic composition (TAnt, and tannins). The highest TAnt values were observed in the intermediate sizes $(25$ and $50 \mathrm{~L})$, whereas for tannins content the highest values were found in the greater tanks $(50$ and $100 \mathrm{~L})$ in both varieties. One of the most relevant effects that tank size could have from the research point of view is increasing or decreasing the variability of the composition of the wine obtained from replicates. When the coefficients of variation (CV) obtained for each variable, tank size and variety are compared (Fig $2 \mathrm{a}$ and $2 \mathrm{~b}$ ), all values were low, especially for $\mathrm{ABV}, \mathrm{pH}$ and TA $(\mathrm{CV}<4 \%)$, but also for TAnt and tannin content $(\mathrm{CV}<8 \%)$. Taking into account that the observed CVs were satisfactory for all tank sizes and varieties (less than 5\%), there is a slightly greater variability in intermediate sizes $(25$ and $50 \mathrm{~L})$ in TE. This fact supports the repeatability of the wines made at any of the tank sizes considered with regards to the major wine composition parameters.

Total anthocyanin composition (Table $5 \mathrm{a}$ and $5 \mathrm{~b}$ ) in the medium size-tanks (25 and $50 \mathrm{~L}$ ) was higher than any other volumes (10 and $100 \mathrm{~L}$ ) in TE. Malvidin glucosides $(\mathrm{G})$ proved much more extracted (up to one third) than the acetyl glucosides (AG). Furthermore, the latter showed almost the same concentration of coumarylglucosides (GC). In CS, the greatest anthocyanin contents were found in the biggest volumes (Table 9), CS tanks of 10 and $25 \mathrm{~L}$ showed delayed extraction of anthocyanins (table 9), giving 117.7mg/L 
of total anthocyanins in $10 \mathrm{~L}$ and $128.5 \mathrm{mg} / \mathrm{L}$ in $25 \mathrm{~L}$ and $361.9 \mathrm{mg} / \mathrm{L}$ in $100 \mathrm{~L}$ and $384.4 \mathrm{mg} / \mathrm{L}$ in $50 \mathrm{~L}$. Thus, in the case of CS it appears that, the larger the tank, the greater the extraction $(50,100)$. In CS, the difference between glycosides $(\mathrm{G})$ and acetyl glucosides (AG) total concentration was not remarkable, with lower extractions observed in the smaller volumes for them all. Reproducibility in terms of anthocyanin content can be said to be satisfactory, since CVs for all anthocyanin families were below $20 \%$, the median CV being $13 \%$ for CS and 10\% for TE (Figure 2c and 2d). Tank size appeared to affect reproducibility, although the observed effect was different for each variety. In TE, the lowest CVs were found for 100 and 25 $\mathrm{L}$ tanks, whereas in CS this occurred in 10 and $50 \mathrm{~L}$ tanks.

Variability in procyanidins content (Figure 2e and 2f) was relatively similar to that observed for anthocyanins; median value was just $9 \%$ except for CS-25 and TE-50 (12-22\%, respectively). CV lower and upper values ranged between $10-22 \%$ in $\mathrm{CS}$ and $5-17 \%$ in TE, dimers showing the higher CV, which indicates that reproducibility was in general terms very satisfactory, particularly in TE, where almost always was below 10\%. In both CS and TE, the lower CVs were associated to $10 \mathrm{~L}$ and $100 \mathrm{~L}$ volumes. In general, the effect of tank size on procyanidins content repeatability was less relevant than it was for anthocyanins.

Comparison with commercial volume

Principal component analyses allowed condensing the information provided by all the analysis variables included in the study into a reduced number of components with a minimum loss of information in both varieties. Thus, in CS, the first component accounted for $44.6 \%$ of variability, the second one for $36.6 \%$ and the third one for $5.9 \%$ (Figure 3a), whereas in TE they were 44.0, 27.3 and $7.2 \%$ respectively (Figure $3 \mathrm{~b})$. In both varieties, the first component included mainly anthocyanin-content variables, the second one procyanidins-content variables, and the third one was linked mainly to acidity ( $\mathrm{pH}$ in $\mathrm{CS}$, and titratable acidity in TE).

PCA scores for all small-scale tanks, average scores for each small-scale volume and commercial scale tank scores are represented in Figure 3a and 3b for CS and TE, respectively. For both varieties, the composition of the wine obtained in $100 \mathrm{~L}$ tanks was clearly more similar to commercial scale wine for the main (first) component, related to anthocyanin content. For the second component, related to procyanidins, wines obtained in 10 and $100 \mathrm{~L}$ volumes were the most similar to commercial scale in CS, whereas in TE differences were smaller in this axis, $10 \mathrm{~L}, 25 \mathrm{~L}$ and $100 \mathrm{~L}$ showing similar scores for this component to that in the commercial scale wine (Figure $3 \mathrm{c}$ and $3 \mathrm{~d}$ ). 

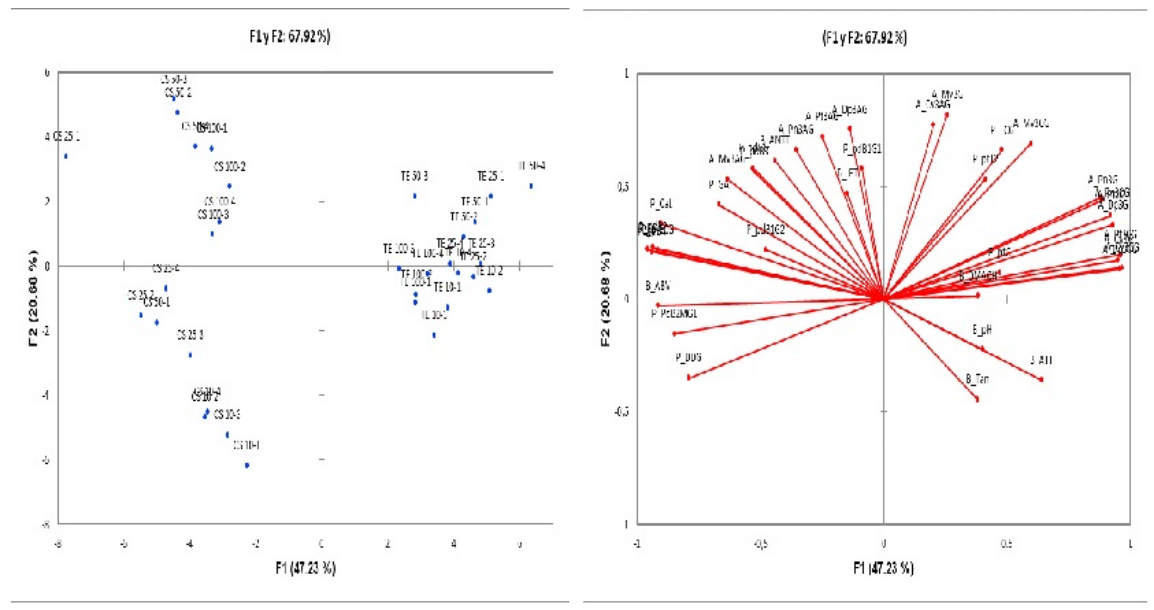

Figure 3: Contribution of wine composition variables to Principal Component Analysis dimensions 1 and 2 in all the small-scale fermentations. The resulting components from this transformation shows that the first two principal component have the highest variance and accounts for as most of the variability in the data. thefirst 2 components contribute to $67.92 \%$ of the total variance. Choosing two components is good enough to show that the two grape and small-scale fermentations are well separated. This justifies that we do a separate analysis of the main components of each variety.

\section{Discussion}

Although other studies with microscale fermentation have shown results using much smaller volumes (1L), our contribution focuses in the relevance of volume fermentation size even when bigger volumes are considered. With regards to repeatability, all tank sizes proved to be adequate, since CV values were in general low. Certain trend was observed to have increased variability in 25 and $50 \mathrm{~L}$ tanks, but differences were small and the CVs obtained were very satisfactory (usually below $15 \%$ when determining phenolics and $5 \%$ on grape and wine composition). This is a very important result, since one of the main concerns of researchers in viticulture and oenology is that reducing tank size in their experiments can increase variability in the fermentation stage, and therefore obtaining less reliable results. According to our data, decreasing tank size from 100 to $10 \mathrm{~L}$ does not cause an increase in variability and, therefore, the reliability of the results is very good.

However, having similar reliability in terms of variability does not mean that tank size does not affect wine representativeness. For both varieties, we observed that the biggest volume was more representative of commercial scale fermentation, particularly for anthocyanins (first component in PCA). Thus, $10 \mathrm{~L}$ tanks achieved the lowest concentration of anthocyanin and phenol extraction into the wine, with the extraction of non-acylated anthocyanins being benefited. De Villiers et al. (2004) found that non-acylated glycosides are more easily extracted, followed by acetyl glycosides and p-coumaroylated, being the latter the more difficult to extract from grape to wine. On the other side, procyanidins, included majorly in the second component of PCA, were extracted in larger quantities in the commercial size-tank, although $10 \mathrm{~L}, 25 \mathrm{~L}$ and $100 \mathrm{~L}$ showed similar scores for this component compared to the commercial scale wine. The pump-overs and the extended maceration made in the commercial wine may affect differently than the gently hand punched down made in the small-scale, due to an additional mechanical action of the pump which is not applicable to small volumes, and leads to a much greater concentration of monomers into the wine. However, despite different extraction of monomers, dimers and trimers, the total procyanidin content is much similar between tanks than that observed in the extraction of anthocyanins.

Tank size affected fermentation dynamics in both varieties, the effects being clearer in CS tanks, where 
fermentation took place more slowly due to the smaller berry size type variety. In both varieties, the smallest tank (10 L) fermented the fastest, no differences being found between the remaining 3 sizes in TE, and being gradually slower as tank size increased in CS. However, tank size did not affect the total time required to complete fermentation (Figure 1).

All in all, according to our results, the smallest tank size used in this study could be representative enough when the goal of winemaking is to compare different field or winemaking strategies (i.e., viticulture practices or yeast trials), as variability was not affected by tank size. Nevertheless, when the objective of small scale winemaking is to perform the wine extraction and phenolic composition, mainly for red phenolic varieties, increasing tank volume (up to $100 \mathrm{~L}$ ) would be necessary to obtain comparable results to commercial scale wines.

\section{Conclusions}

Small-scale winemaking represents a necessary and valuable tool for viticulture and oenological research, although small size tanks should be only used when the objective of research is to compare different field or winery treatments in relative terms. Instead, to define the phenolic composition of a commercial wine, bigger volumes are needed to produce wines with similar properties and phenolic content.

\section{Acknowledgements}

Funded by National Projects CICYT Ref. AGL 2008-04525-CO2-O2); CICYT Ref: AGL2011-30408-CO4$02 .^{9}$

\section{References}

(1) Copigmentation in Model Wine Solution: Occurrence and Relation to Wine Ageing.. American Journal of Enology and Viticulture 1999, 50:211-218..

(2) González-Manzano, S.; Rivas-Gonzalo, J. C.; Santos-Buelga, C. Extraction of Flavan-3-Ols from Grape Seed and Skin into Wine Using Simulated Maceration. Analytica Chimica Acta 2004, 513 (1), 283-289. https://doi.org/10.1016/j.aca.2003.10.019.

(3) Lopes, C. A.; Broock, M. van; Querol, A.; Caballero, A. C. Saccharomyces Cerevisiae Wine Yeast Populations in a Cold Region in Argentinean Patagonia. A Study at Different Fermentation Scales. Journal of Applied Microbiology 2002, 93 (4), 608-615. https://doi.org/10.1046/j.1365-2672.2002.01738.x.

(4) Santesteban, L. G.; Miranda, C.; Royo, J. B. Influence of the Freezing Method on the Changes That Occur in Grape Samples after Frozen Storage. Journal of the Science of Food and Agriculture 2013, 93 (12), 3010-3015. https://doi.org/10.1002/jsfa.6133.

(5) Casalta, E.; Sablayrolles, J.-M.; Salmon, J.-M. Comparison of Different Methods for the Determination of Assimilable Nitrogen in Grape Musts. LWT - Food Science and Technology 2013, 54 (1), 271-277. https://doi.org/10.1016/j.1wt.2013.05.009.

(6) Schmid, F.; Schadt, J.; Jiranek, V.; Block, D. E. Formation of Temperature Gradients in Large- and Small-Scale Red Wine Fermentations during Cap Management. Australian Journal of Grape and Wine Research 2009, 15 (3), 249-255. https://doi.org/10.1111/j.1755-0238.2009.00053.x.

(7) Gordillo, B.; Lo

pez-Infante, M. I.; Rami 
rez-Pe

rez, P.; Gonza

lez-Miret, M. L.; Heredia, F. J. Influence of Prefermentative Cold Maceration on the Color and Anthocyanic Copigmentation of Organic Tempranillo Wines Elaborated in a Warm Climate. Journal of Agricultural and Food Chemistry 2010, 58 (11), 6797-6803. https://doi.org/10.1021/jf100084x.

(8) Nadal, M. Phenolic Maturity in Red Grapes. In Methodologies and Results in Grapevine Research; Springer Netherlands, 2010; pp 389-409. https://doi.org/10.1007/978-90-481-9283-0_28.

(9) Lasanta, C.; Caro, I.; Gómez, J.; Pérez, L. The Influence of Ripeness Grade on the Composition of Musts and Wines from Vitis Vinifera Cv. Tempranillo Grown in a Warm Climate. Food Research International 2014, 64, 432-438. https://doi.org/10.1016/j.foodres.2014.07.039.

(10) Casalta, E.; Aguera, E.; Picou, C.; Rodriguez-Bencomo, J. J.; Salmon, J. M.; Sablayrolles, J. M. A Comparison of Laboratory and Pilot-Scale Fermentations in Winemaking Conditions.. Appl Microbiol Biotechnol 2010, 87, 1665-1673.

(11) Redirecting.

(12) Determination of Anthocyanins in Wine by Direct Injection Liquid Chromatography-Diode Array Detection-Mass Spectrometry and Classication of Wines Using Discriminant Analysis.. Journal of Chromatography A 2004, 1054, 195-204.

(13) Frozen Grape Research Finds Warm Reception in the Wine Industry: a Novel Winemaking Process and Posible Strategy for Dealing with Surplus Grapes.

(14) Lopes, C. A.; van, B. M.; Querol, A.; Caballero, A. C. Saccharomyces Cerevisiae Wine Yeast Populations in a Cold Region in Argentinean Patagonia. A Study at Different Fermentation Scales.. J Appl Microbiol 2002, 93, 608-615.

(15) Influence of the Freezing Method on the Changes That Occur in Grape Samples after Frozen Storage.

(16) Formation of Temperature Gradients in Large- and Small-Scale Red Wine Fermentations during Cap Management.

(17) Maxwell, O. I.; Chinwuba, U. B.; Onyebuchukwu, M. G. Protein Enrichment of Potato Peels Using Saccharomyces Cerevisiae via Solid-State Fermentation Process. Advances in Chemical Engineering and Science 2019, 09 (01), 99-108. https://doi.org/10.4236/aces.2019.91008. 